\title{
REPERCUSIÓN ECONÓMICA POR CAMBIO DE USO Y PROPIEDAD DEL AGUA EN EL LITORAL ORIOLANO
}

\author{
Cipriano Juárez Sánchez-Rubio \\ José Fernando Vera Rebollo
}

*Este trabajo ha sido realizado dentro de los planes de investigación de la Comisión Asesora de Investigación Científica y Técnica.

\section{INTRODUCCIÓN}

La idea de trasvasar agua a las provincias de Alicante, Almería y Murcia es antigua pero no cristaliza hasta la ley del «Aprovechamiento conjunto del trasvase Tajo-Segura» de 1971. De $\operatorname{los} 1.000 \mathrm{hm}^{3} /$ año a trasvasar ninguna cantidad era para atender la demanda urbana. Pero las precisiones de Lorenzo Pardo y las promulgadas por la citada ley distan mucho de la realidad, ya que «las necesidades turísticas e industriales, el aumento de la población y el consumo mayor de agua por habitante y día convierten la llegada de los caudales al agro en poco menos que secundario» ${ }^{1}$.

Este desplazamiento de uso desde la agricultura, en la que se obtiene un valor añadido bajo por agua embalsada, a la industria y servicio con un valor añadido mucho más alto, ha obligado a que la ley de 1980 contemple que el 40\% del caudal trasvasado se destinará a uso urbano e industrial. Sin embargo, las comunidades autónomas de Castilla-La Mancha y Extremadura quieren sustituir por una nueva ley el término de «Aprovechamiento conjunto del trasvase Tajo-Segura» por el de «Transferencia de recursos hidráulicos de la cuenca del Tajo a la cuenca del Segura». El cambio de denominación encierra la pretensión de convertir en voluntaria la cesión de los caudales del Tajo al Segura según acuerdos o convenios intercomunitarios que se acuerden en cada estatuto de autonomía.

Ante esta situación y previendo los enfrentamientos que se pueden producir entre las diferentes comunidades autónomas, el Estado asumirá, según especifica el borrador de la nueva Ley de Aguas, el control de las cuencas de todo el [140] territorio nacional. En consecuencia, el agua seguirá trasvasándose y su volumen dependerá de las necesidades de los sectores productivos que ofrecen mayor rentabilidad.

Un análisis comparativo entre la capacidad de los embalses construidos, la potencia instalada en los mismos y el número de hectáreas ocupadas por el riego pone de manifiesto el continuado desequilibrio entre el volumen de agua embalsada y su aprovechamiento diferente. Así, la relación entre el incremento de superficie transformada y los hectómetros cúbicos embalsados demuestran que tales valores han sido muy diferentes en el tiempo puesto que entre 1900 y 1940 es de $53 \mathrm{ha} / \mathrm{hm}^{3}$, entre 1940-50 de 24, entre 1950-60 de 28 y entre 1960-79 de $42 \mathrm{ha} / \mathrm{hm}^{3}$. En cambio, la potencia hidroeléctrica instalada ha pasado de 1.350 millones de $\mathrm{Kw}$ $(100 \%)$ en 1.940 a 13.504 millones de kilowatios, 1.000, 3\%, en 1978. Se puede decir que en España no ha existido una política hidráulica tendente a la transformación en regadío, sino una política con fines más lucrativos como la producción hidroeléctrica y el de asegurarse, mediante trasvase, el abastecimiento de agua a los centros industriales y turísticos del sureste peninsular» ${ }^{2}$.

Este cambio en el uso preferente del agua fue posible por la propia regulación que sobre

\footnotetext{
${ }^{1}$ MORALES, A., y JUÁREZ, C., «Cambios en el uso del agua», Estudios Geográficos, 1981, N. ${ }^{\text {1 }}$ 165, pág. 389.

${ }^{2}$ MORALES, A., y JUÁREZ, C., Ob. cit., pág. 381.
} 
la gestión y administración hace la Ley de Aguas de 1879 . Como consecuencia, del $42 \%$ de las tierras cultivadas del país, sólo el 14\%, la tercera parte, están ocupadas por el regadío. Pero esta superficie transformada se ha conseguido porque además de la utilización de las aguas procedentes de los cauces fluviales se han explotado los caudales subterráneos del valle del Ebro, cuenca mediterránea y ambas castillas. Es así como en 1974 la superficie total regada era de 2.584.000 ha, de las que 620.000, el 24\%, utilizaban agua subterránea. En 1981 la cantidad se eleva a 2.853 .000 ha, de las cuales el 33\% están abastecidas por caudales subterráneos. Este incremento es producto de la iniciativa del capital privado y de una serie de decretos sobre el fomento del regadío. Pero al no existir ordenación jurídica alguna que regule estas captaciones, se producen superposiciones, sobreexplotación, salinización y agotamiento de los pozos y, consecuentemente, repercusiones en los cultivos al disminuir la rentabilidad.

La carencia de recursos hídricos condiciona una gran parte de las actividades humanas y sus disponibilidades constituyen un potencial básico para el desarrollo económico. En su apoyo «basta señalar que en España la demanda actual de agua para regadíos es de $21.526 \mathrm{hm}^{3} / \mathrm{año}$, de los que el $23 \%$ son extraídos de los acuíferos subterráneos y el resto de los cauces superficiales. Las demandas para abastecimiento urbano, con dotaciones medias de 200-300 litros por habitante y día, alcanza $3.500 \mathrm{hm}^{3} /$ año de los que el $37 \%$ proceden del bombeo en pozos y sondeos. La demanda de agua para la industria llega a $9.000 \mathrm{hm}^{3} /$ año, aunque este dato no está actualizado» ${ }^{3}$. En estos momentos [141] la participación de las aguas subterráneas en la demanda total del país era del 23\%. En la actualidad (1983), se afirma que las aguas subterráneas abastecen el $40 \%$ de las necesidades de los núcleos urbanos del país y el $30 \%$ de las necesidades totales si se incluye el consumo industrial de agua. Sin embargo, las desviaciones regionales con respecto a los porcentajes indicados son muy grandes «ya que en las cuencas hidrográficas del Guadiana, Júcar y Sur las aguas subterráneas suponen más del 60\% del abastecimiento urbano e industrial» ${ }^{4}$.

En consecuencia, y conociendo el anteproyecto de la nueva Ley de Aguas cuyo objetivo es la socialización del recurso, es obligado el estudio de una zona donde la incidencia económica y social afectará muy considerablemente.

\section{REPERCUSIONES EN EL SECTOR AGRARIO}

Con la idea de valorar la incidencia socioeconómica que la futura Ley de Aguas va a tener en el sector agrario, se ha delimitado una zona con una agricultura de regadío altamente capitalizada, el litoral oriolano y el campo de La Horadada, cuyos regadíos son la consecuencia de un constante proceso de cribado de mantos hipogeos, que se ha visto completado y potenciado con la llegada de las aguas del Tajo.

Hasta los primeros años del decenio de 1960, momento en el que la extracción del agua subterránea se generaliza e intensifica, la zona referida se caracteriza por el predominio de una agricultura de secano, cuyos aprovechamientos dominantes eran la arboricultura (olivo, algarrobo y almendro) y la cerealicultura (cebada y trigo), a excepción de algún pequeño rodal de regadío mantenido por las antiguas norias; la avena y algunas leguminosas completaban el cuadro de cultivos y la cosecha estaba condicionada por entero a la pluviometría. A la aridez como limitación física se unían otros problemas estructurales, de entre los cuales, sin duda, el más importante era el reparto de la propiedad, todo lo cual determinaba una corriente de

${ }^{3}$ GARCÍA-LOYGORRI, A., «Las aguas subterráneas en la ordenación territorial», Boletín Geológico y Minero, 1979, t. XC-IV, pág. 70.

${ }^{4}$ FONTANILLA SORIANO, P., «Gestión y conservación de los acuíferos como consecuencia del Programa Nacional de Investigación de Aguas Subterráneas», Boletín Geológico y Minero, 1976, t. LXXXVII, págs. 57-71. 
emigración y escaso crecimiento poblacional (en 1940 El Pilar de La Horadada contaba con 1.433 habitantes) $)^{5}$.

A la hora de evaluar los recursos hídricos en el sector referido se ha de establecer una diferenciación básica entre recursos tradicionales y nuevos. El aprovechamiento, mediante un sistema de boqueras de las aguas que esporádicamente circulan por los cauces de las ramblas contribuían a paliar el tradicional déficit hídrico. Pero el recurso tradicional más importante lo constituyen [142] las aguas subterráneas, captadas en un primer momento a escasa profundidad mediante norias y cenias y, posteriormente, en función de la mayor potencia eléctrica instalada, con motobombas, lo cual hizo posible la explotación de acuíferos más profundos. Merced a las mayores disponibilidades de caudales se produjo un cambio en los aprovechamientos agrarios, causa, a la vez, de una nueva dinámica demográfica y económica.

A partir de 1979, la llegada de las aguas del Tajo, con la entrada en funcionamiento del Canal del Campo de Cartagena, permite a la zona contar con un nuevo recurso hídrico, capaz de mejorar los regadíos tradicionales, transformar secanos y ampliar la superficie cultivada, y que además tiene carácter público, frente a las aguas subterráneas explotadas particularmente por los agricultores. Este hecho conviene tenerlo muy en consideración, ya que el aprovechamiento de un mismo acuífero por gran número de agricultores ha conducido a una sobreexplotación y salinización de los mantos al no existir ninguna norma que regule y racionalice las extracciones. Frente a esta situación las aguas foráneas, cuando llegan, son administradas y distribuidas por la Confederación Hidrográfica a través de Comunidades de Regantes y Sociedades Agrarias de Transformación, creadas a tal efecto, en las cuales se integran los agricultores para conseguir asignaciones de caudales. Asimismo, el precio y la calidad de dichas aguas superficiales hacen aconsejable su uso frente a las subterráneas. Pero las escasas dotaciones del trasvase en los últimos años obligan a recurrir al uso del agua de pozo.

Actualmente, ante las expectativas de la futura Ley de Aguas, este proceso presenta una incidencia particular en función del tipo de explotación afectada. En líneas generales, la cuestión se centra en el problema de las inversiones realizadas para la extracción de las aguas subterráneas y el mantenimiento de las superficies productivas, lo cual asegura la permanencia de los ingresos económicos, con una indudable trascendencia en base al acusado predominio de las explotaciones familiares.

Las grandes explotaciones (mayores de $100 \mathrm{ha}$ ), tres en toda el área objeto de estudio, han realizado grandes inversiones para asegurarse unos caudales mínimos que les permitieran la transformación en regadío y su mantenimiento. Como caso paradigmático tenemos el de la explotación agrícola Lo Romero (1.025 ha) donde se construyeron trece pozos a fines del decenio de 1950 y principios del de 1960, los cuales costaron cada uno un millón de pesetas, incluidas las bombas, para la transformación a regadío de secanos y montes. En la actualidad sólo funcionan dos sondeos cuyos aforos son de $90 \mathrm{~m}^{3} /$ hora y $20 \mathrm{~m}^{3} /$ hora, mientras el canal del Campo de Cartagena (trasvase Tajo-Segura), sólo afecta a las sesenta ha de la finca que quedan por debajo de la cota de $70 \mathrm{~m}$, es decir, en la margen izquierda del referido canal (la explotación está integrada en la S.A.T. «La Pedrera»). Todo ello ha motivado la necesidad de comprar caudales de pozos vecinos (a $15 \mathrm{pts} . \mathrm{m}^{3}$ ), muchos de ellos propiedad de agricultores familiares que los mantienen como reserva para ser utilizados cuando las aguas del canal no llegan o son insuficientes, por lo que resulta obvio que en los momentos de escasez en el trasvase la situación se agrave [143] particularmente en Lo Romero al faltarle las dotaciones de caudales subterráneos vecinos. Al objeto de almacenar agua en previsión de situaciones de escasez se han construido dos embalses con una capacidad de 700.000 y $100.000 \mathrm{~m}^{3}$, a la vez que se generalizan los

\footnotetext{
${ }^{5}$ VERA REBOLlO, J. F., Tradición y cambio en el campo del Bajo Segura, Alicante, Instituto de Estudios Alicantinos (en prensa).
} 
métodos que permiten una mayor economía del agua (goteo).

Otro caso de gran explotación en el campo de La Horadada es el de La Carrasca, con unas 237 ha que se reparten entre 60 de alcachofas, 18 de invernaderos, 12 de melones, 12 de guisantes, 24 de forrajeras y resto en barbecho. Cuenta para el riego con cuatro perforaciones cuyo aforo total es de $300 \mathrm{~m}^{3} /$ hora y fue precisamente en esta hacienda donde se inició el aprovechamiento intensivo de las aguas hipogeas de la zona, pero en la actualidad se observa un impresionante descenso del nivel piezométrico.

En otras grandes explotaciones, el carácter agroturístico de las mismas ha determinado que una parte sustancial del agua subterránea se destine al abastecimiento del área urbanizada, en detrimento de los regadíos, como se constata en La Dehesa de Campoamor (927 ha de las que 113 son de regadío), que cuenta con 9 pozos entre 80 y $100 \mathrm{~m}$ de profundidad que extraen en total unos 8.100 litros/minuto, con los cuales se abastece la urbanización y el área de cultivos, si bien el agotamiento de los mantos hace pensar en la necesidad de fuentes de aprovisionamiento alternativas (trasvase).

Las inversiones en perforaciones se han realizado también a nivel de medianas explotaciones aunque éstas son escasas, por lo que resulta de mayor interés el caso de los agricultores familiares, los más abundantes en toda la zona. En este sentido, lo primero que debe tenerse en cuenta es que este tipo de explotaciones proceden en buena parte de la parcelación realizada a fines del decenio de 1950 y principios de 1960 en antiguas haciendas de secano las cuales, a lo sumo, contaban con un sondeo para toda su extensión. En efecto, en los dos casos más significativos de parcelaciones. La Pacheca de Abajo y Torre Segura se constata la existencia de una perforación antigua que pasó a manos de los nuevos propietarios, agricultores familiares, para su aprovechamiento conjunto. En Torre Segura el primer pozo se agotó y los agricultores construyeron otro que costó unas 300.000 pesetas a principios de los años sesenta. Ante las expectativas del trasvase constituyeron la S.A.T. número 9.032 y continuaron con nuevos alumbramientos. Actualmente, el riego se realiza mediante dos pozos artesianos $(300 \mathrm{~m}$ de profundidad) cuyo caudal es de $40 \mathrm{~m}^{3} /$ hora, y otro de aguas comunes que extrae $45 \mathrm{~m}^{3}$ en las cinco horas que la bomba puede trabajar cada día, ya que para evitar una rápida salinización es necesario limitar la extracción. El trasvase les ha suministrado $87.000 \mathrm{~m}^{3}$ a lo largo de $1982 \mathrm{de}$ una dotación pedida de 600-700.000 $\mathrm{m}^{3}$. Los aprovechamientos de las explotaciones familiares agrupadas en esta S.A.T. suman en conjunto unos $400.000 \mathrm{~m}^{2}$ de invernaderos, 200 tahúllas de cítricos (1 tahúlla: $\left.1.185 \mathrm{~m}^{2}\right)$ y unas 400 tahúllas de alcachofa y melón, es decir, cultivos intensivos y con grandes necesidades de agua.

La mutación de los paisajes agrarios está totalmente condicionada por el [144] elemento agua, ya que su escasez determina que se beneficien unos cultivos (cítricos) y la sustitución de otros (alcachofa).

En La Pacheca de Abajo los agricultores familiares (propiedad media 8 tahúllas) se constituyeron en Grupo Sindical de Colonización, luego S.A.T. 1.380, y contaban en el momento de la parcelación (fines del decenio de 1950 ) con un pozo de aguas comunes $(70-80 \mathrm{~m})$ pero fue necesario realizar otro sondeo cuyo aforo era de $60 \mathrm{~m}^{3} /$ hora y que extrajo agua durante 8 ó 10 años; al agotarse éste fue necesario realizar otra perforación $(160 \mathrm{~m})$ que dio un aforo de 70 $\mathrm{m}^{3} /$ hora y que costó un millón de pesetas, para lo que dispusieron de un préstamo del INC por 25 años. Los socios tienen establecida una cuota de 4.000 pesetas por parcela y año y pagan el agua a 8 pesetas $/ \mathrm{m}^{3}$ para hacer frente a los cuantiosos gastos de extracción (sólo de fluido eléctrico se vienen pagando unas $200.000 \mathrm{pts} . / \mathrm{mes})$.

Todo esto pone de relieve el esfuerzo que el agricultor ha debido realizar para transformar sus tierras a regadío, en base a la iniciativa privada en la casi totalidad de los casos, y agrupados a veces en Grupos o Sociedades. Tras unos inicios difíciles, en los cuales el agricultor vive entrampado con las entidades de crédito (casi la totalidad de cajas rurales de 
Alicante y Murcia y varios bancos importantes tienen establecidas sucursales en El Pilar, lo que da idea de la importancia del crédito en esta agricultura) se consigue un elevado nivel de renta que permite vivir a la familia campesina con una superficie de unas 8 tahúllas (alrededor de 1 ha) dedicadas al cultivo intensivo y, sobre todo, al invernadero.

Las necesidades de mano de obra de los nuevos cultivos han determinado una fuerte corriente inmigratoria en los dos últimos decenios, con la llegada de jornaleros de la vecina Murcia o de provincias meridionales, los cuales contribuyen eficazmente al espectacular auge poblacional que se observa: en 1970 El Pilar de La Horadada contaba con 3.698 habitantes y en 1981 ya eran 5.130, con un 30,5\% de inmigrados, lo que da idea de las repercusiones del cambio experimentado en la agricultura.

La limitación de los caudales subterráneos tendría graves consecuencias sobre tres conceptos esenciales: empleo, renta y consumo, por lo que se hace necesaria una racionalización de las extracciones como única garantía de que los mantos no se van a agotar y salinizar por sobreexplotación, lo que supondría caer en una total dependencia del agua foránea, a todas luces insuficiente para mantener las superficies productivas, lo cual motivaría una grave crisis en el sector.

Desde el punto de vista de la trascendencia social, la puesta en regadío de extensas áreas en la zona ha sido la causa de un importante aumento del nivel de empleo y renta, con la consiguiente influencia en el nivel de consumo de la población. El predominio de las explotaciones familiares es un factor esencial para entender las repercusiones sociales que ha suscitado la transformación de la agricultura. La práctica totalidad de la población agraria de este espacio ha experimentado el aumento de los niveles de renta y son muchos los jornaleros [145] inmigrados que han podido acceder a la propiedad de la tierra. En este sentido, reviste gran interés el hecho de que la escasez de agua esté determinando una nueva orientación productiva que beneficia unos cultivos muy poco exigentes en mano de obra y altamente tecnificados en cuanto a los sistemas de laboreo, como es el caso de los cítricos, de forma que el regadío deja de contribuir a una transformación social en el sentido de creación de empleo y mayor nivel de renta y consumo para la clase jornalera y produce una transformación esencialmente técnica y de alta rentabilidad económica, pero que consolida la estructura social existente, tal y como viene ocurriendo en algún área próxima (San Miguel de Salinas). Aun así, el regadío es rentable socialmente por el empleo que genera y económicamente, ya que sus cultivos permiten ingresar divisas (la exportación agraria nacional en 1981 fue de 370.802,1 millones de pesetas, de los que la provincia de Alicante contribuyó con 55.397,83, es decir, el 14,94\%.

En resumen tres factores esenciales, agua, sol y capacidad de iniciativa han actuado como motor para convertir un espacio agrario de escasa importancia económica en un emporio de riqueza. Pero los puestos de trabajo y los ingresos económicos que genera la agricultura de regadío están condicionados a una política de racionalización en la explotación de los escasos recursos de aguas subterráneas, si bien ello no debe suponer la reducción de los caudales para cada explotación y la disminución de la superficie productiva, sobre todo si los regadíos responden a los esfuerzos particulares del agricultor. Se impone a la vez la necesidad de difundir los modernos procedimientos de riego localizado, que permiten un ahorro importante de caudales. La implantación de tales sistemas deberá llevarse a cabo con una mayor información al agricultor sobre la utilidad y economía de tales métodos por parte de los organismos dependientes del Ministerio, así como de una política de créditos al sector.

\section{INCIDENCIA EN EL SECTOR SERVICIOS}

La nueva Ley de Aguas puede tener unas repercusiones importantes en las actividades turísticas por lo que respecta al abastecimiento de las urbanizaciones y de los núcleos turísticos 
en general, dado que éstos son grandes consumidores de agua.

Las urbanizaciones de la zona costera oriolana, ubicadas en lo que era espacio agrario, han debido contar con sondeos para garantizar el abastecimiento a la población veraneante, lo cual se hizo a expensas de los caudales destinados en primer momento a la agricultura. Desde el decenio de 1960 pareció que era más rentable regar metros cuadrados de urbanización que tahúllas de cultivo. Fue así como los pozos se pusieron al servicio de las urbanizaciones y en las fincas donde se mantuvo el carácter agroturístico, el agua se concedió prioritariamente a la urbanización, con el agravante de que se produce una coincidencia entre la época de mayor afluencia de veraneantes y las mayores necesidades hídricas de los cultivos. En algún caso la superficie de cultivo llegó a desaparecer por completo, como ocurrió en la urbanización [146] La Zenia; en otros, como en Campoamor, la abundancia de aguas subterráneas aún permite regar los cultivos y abastecer a la urbanización. Pero en los últimos años, el crecimiento de las urbanizaciones se ha venido acompañando de una sobreexplotación de los mantos hipogeos, agotamiento y proceso creciente de salinización, lo cual ha determinado la constitución de la Asociación de Urbanizadoras de las Playas de Orihuela, con la idea de conseguir asignaciones de agua del trasvase Tajo-Segura para uso turístico, a pesar de que el canal ya se muestra insuficiente para atender a los nuevos regadíos. El hecho de que la actividad constructora no se haya detenido, a pesar de los problemas de abastecimiento de agua, hace pensar que la asignación de caudales del trasvase a las urbanizaciones es una realidad inmediata, con lo que el problema de la falta de agua en la agricultura se verá aún más agravado, a no ser que se aumenten las dotaciones del trasvase.

Las urbanizaciones han debido recurrir a la compra del agua subterránea a particulares, auténticos especuladores, ya que son propietarios de unas parcelas que dejan sin cultivar y que destinan únicamente a la explotación intensiva de los acuíferos, con el consiguiente peligro de agotamiento y salinización de las aguas, cuyo precio oscila entre las 12 y 15 pts. $/ \mathrm{m}^{3}$.

La urbanización Cabo Roig compra unos $1.000 \mathrm{~m}^{3} /$ día en verano para el abastecimiento de unos 400 chalés que albergan alrededor de 2.500 personas. La vecina urbanización La Zenia (C.I.T.) cuenta con tres pozos propios que aforan 25 litros/sg. por lo que también recurre a la compra del agua a un particular que tiene cinco pozos con un aforo medio de 20 litros/seg.; en verano la urbanización, con 700 viviendas y unos 3.500 habitantes, consume alrededor de 1.500 $\mathrm{m}^{3} /$ día y se ha iniciado una segunda fase de urbanización, lo que hace suponer un considerable aumento de las necesidades de agua. Una situación semejante se constata en el resto de las urbanizaciones, con un consumo medio en la temporada alta por habitante y día de unos 400 litros, lo que da idea del despilfarro de agua que se produce en las urbanizaciones.

\section{CONCLUSIÓN}

Como consecuencia de todo este proceso, resulta imprescindible una planificación para utilizar de forma ordenada las aguas dulces. Tal ordenación exige olvidarse del régimen de propiedad entre las aguas subterráneas y superficiales. Asimismo, es conveniente la adopción de una serie de medidas complementarias de las cuales la principal sería la revisión y actualización de la Ley de Aguas de 1879, en orden a declarar el carácter público de todas las aguas. En esta línea de socialización se manifiesta el borrador de la futura Ley de Aguas cuando dice que todas las aguas continentales del territorio español, tanto superficiales como subterráneas, están subordinadas al interés general y constituyen un recurso natural unitario de dominio público estatal.

No obstante, y según el borrador de la nueva ley, los agricultores no se verán privados de sus derechos, sino obligados a racionalizar las extracciones de acuerdo con planes concretos para cada cuenca hidrográfica. Con estas [147] medidas se pretende evitar la sobreexplotación 
abusiva de los acuíferos que conduce a la pérdida de calidad del agua en las zonas adyacentes, la especulación del agua como bien escaso y la salinización de acuíferos próximos al mar o en el interior del país, también por sobreexplotación. 